\title{
Feasibility and efficacy of helical intensity-modulated radiotherapy for stage III non-small cell lung cancer in comparison with conventionally fractionated 3D-CRT
}

\author{
Jian He, Yan Huang, Yixing Chen, Shiming Shi, Luxi Ye, Yong Hu, Jianying Zhang, Zhaochong Zeng \\ Department of Radiation Oncology, Zhongshan Hospital, Fudan University, Shanghai 200032, China \\ Contributions: (I) Conception and design: Z Zeng, J He; (II) Administrative support: Z Zeng, J He; (III) Provision of study materials or patients: Z \\ Zeng, J He; (IV) Collection and assembly of data: Y Chen, Y Huang, S Shi; (V) Data analysis and interpretation: Y Huang, S Shi; (VI) Manuscript \\ writing: All authors; (VII) Final approval of manuscript: All authors. \\ Correspondence to: Zhaochong Zeng, MD. Department of Radiation Oncology, Zhongshan Hospital Affiliated to Fudan University, Shanghai 200032, \\ China. Email: zeng.zhaochong@zs-hospital.sh.cn.
}

Background: The standard treatment for stage III non-small-cell lung cancer (NSCLC) is still $60 \mathrm{~Gy}$ in conventional fractions combined with concurrent chemotherapy; however, the resulting local controls are disappointing. The aim of this study was to compare and assess the feasibility and efficacy of hypofractionated chemoradiotherapy using helical tomotherapy (HT) with conventional fractionation as opposed to using three-dimensional conformal radiotherapy (3D-CRT) for stage III NSCLC.

Methods: Sixty-nine patients with stage III (AJCC 7th edition) NSCLC who underwent definitive radiation treatment at our institution between July 2011 and November 2013 were reviewed and analyzed retrospectively. A dose of $60 \mathrm{~Gy}$ in 20 fractions was delivered in the HT group (n=34), whereas 60 Gy in 30 fractions in the 3D-CRT group $(n=35)$. Primary endpoints were toxicity, overall response rate, overall survival (OS) and progression-free survival (PFS).

Results: The median follow-up period was 26.4 months. V20 ( $\mathrm{P}=0.005)$, V30 ( $\mathrm{P}=0.001), \mathrm{V} 40(\mathrm{P}=0.004)$, mean lung dose $(\mathrm{P}=0.000)$ and max dose of spinal cord $(\mathrm{P}=0.005)$ were significantly lower in the HT group than in the 3D-CRT group. There was no significant difference in the incidences of acute radiation pneumonitis $(\mathrm{RP}) \geq$ grade 2 between the two groups, whereas the incidences of acute radiation esophagitis $\geq$ grade 2 were significantly lower in the HT group than in the $3 \mathrm{D}$-CRT group $(\mathrm{P}=0.027)$. Two-year overall response rate was significantly higher in the HT group than in the 3D-CRT group $(\mathrm{P}=0.015)$. One- and 2-year OS rates were significantly higher in the HT group (95.0\% and 68.7\%, respectively) than in the 3D-CRT group $(85.5 \%$ and $47.6 \%$, respectively; $\mathrm{P}=0.0236)$. One- and 2 -year PFS rates were significantly higher in the HT group (57.8\% and $26.3 \%$, respectively) than in the 3D-CRT group (32.7\% and $11.4 \%$, respectively; $\mathrm{P}=0.0351$ ). Univariate analysis indicated that performance status (PS), $\mathrm{T}$ stage and radiotherapy technique were significant prognostic factors for both OS and PFS. Multivariate analysis indicated that PS and radiotherapy technique were independent prognostic factors of OS and PS was independent prognostic factor of PFS.

Conclusions: Hypofractionated chemoradiotherapy via HT can shorten the radiotherapy time without increasing treatment-related toxicity. The preliminary findings are that OS and PFS can be improved by hypofractionated chemoradiotherapy via HT for patients with stage III NSCLC.

Keywords: Lung cancer; helical tomotherapy (HT); chemoradiotherapy; toxicity; progression-free survival (PFS); overall survival (OS); stage III

Submitted Oct 27, 2015. Accepted for publication Feb 14, 2016.

doi: $10.21037 /$ jtd.2016.03.46

View this article at: http://dx.doi.org/10.21037/jtd.2016.03.46 


\section{Introduction}

Lung cancer was the most frequently diagnosed cancer and the leading cause of cancer death among males in 2012. More than $80 \%$ of lung cancers are non-smallcell lung cancer (NSCLC), one third of which are newly diagnosed, locally advanced NSCLC (1). The current standard approach for treating inoperable, locally advanced NSCLC is concurrent chemoradiotherapy. Patients treated with doses of 60-66 Gy still experienced a local failure rate of approximately $30-40 \%$ in most of the randomized concurrent chemotherapy trials $(2,3)$. For a long time, treatment using higher doses was assumed to outperform conventional radiation therapy using standard doses (4). However, Radiation Therapy Oncology Group (RTOG) 0617 challenged this assumption (5). As reported in RTOG 0617, 74 Gy was not found to increase efficacy, but did increase toxicity compared with 60 Gy using 3-dimensional conformal radiotherapy (3D-CRT) or intensity-modulated radiotherapy (IMRT). One of main reasons for the failure might be that higher doses could not be delivered safely via 3D-CRT due to the proximity of organs at risk (OARs) such as the esophagus, proximal bronchial tree, heart, and spinal cord. As conventional radiotherapy approaches have reached a therapeutic plateau, strategies of treatment intensification attempting to improve local control in stage III NSCLC, such as hypofractionation regimens, shortening overall treatment time, and advanced radiotherapy techniques are on the way (6-9).

Helical tomotherapy (HT) (Accuray Incorporated, Sunnyvale, CA, USA) is an ideal tool for delivering highdose RT without a significant increment in toxicity, because this intensity-modulation system allows for an imageguided radiation therapy (IGRT) (10-12). Compared with non-IGRT for the treatment for tumor, IGRT has enabled innovators in the field to accelerate their exploration of a number of different paradigms of radiation delivery, including toxicity reduction, dose escalation, and hypofractionation (13-15).

To our knowledge, there are few reports comparing the feasibility and efficacy between hypofractionated chemoradiotherapy via HT and conventional fractionated chemoradiotherapy via $3 \mathrm{D}-\mathrm{CRT}$ in patients with stage III NSCLC. Therefore, this study was aimed to compare the feasibility and efficacy between a total dose of $60 \mathrm{~Gy} / 20$ fractions via HT with chemotherapy and $60 \mathrm{~Gy} / 30$ fractions via 3D-CRT with chemotherapy in stage III NSCLC. Primary end points were toxicity, locoregional control, and progression-free survival (PFS). Secondary endpoints were overall response rate and overall survival (OS).

\section{Methods}

\section{Patients}

Patients were required to be $\geq 18$ years old, with an Eastern Cooperative Oncology Group (ECOG) performance status (PS) of 0 to 2 and weight loss $<10 \%$ in the last three months. Patients with unresectable stage III NSCLC were diagnosed histologically or cytologically. Pretreatment evaluation consisted of complete blood count and liver function tests; a computed tomography (CT) scan of the chest and upper abdomen, including the liver and adrenal glands, or liver ultrasound; bone scan, bronchoscopy, a CT or magnetic resonance imaging scan of the brain, pulmonary function tests (PFT). ${ }^{18} \mathrm{~F}$-labeled fluorodeoxyglucose positron emission tomography/CT $\left({ }^{18} \mathrm{FDG}-\mathrm{PET}\right)$ was optional. In this study, all baseline evaluations were performed within four weeks before the beginning of the treatment and PFT were performed at the start. Lung function at the start was required to show at least $40 \%$ of expected value for forced expiratory volume at 1 second $\left(\mathrm{FEV}_{1}\right)$, and corrected diffusing capacity for carbon monoxide (DLCOc) $>50 \%$ predicted. According to Global Initiative for Chronic Obstructive Lung Disease (GOLD), updated 2013, PFT was required to make the diagnosis: the presence of a post-bronchodilator $\mathrm{FEV}_{1} / \mathrm{FVC}<0.70$, confirming the presence of persistent airflow limitation and thus of chronic obstructive pulmonary disease (COPD). A part of patients diagnosed with GOLD 3 COPD $\left(30 \% \leq \mathrm{FEV}_{1}<40 \%\right)$ and all the patients diagnosed with GOLD 4 COPD $\left(\mathrm{FEV}_{1}<30 \%\right)$ were not included. Patients who received previous thoracic radiotherapy, previous surgery, previous chemotherapy with bleomycin, regular use of supplemental oxygen, or patients who received a total radiation dose less than 50 Gy were excluded. Our institutional Medical Ethics Committee approved the treatment protocol, and all patients submitted written informed consent before inclusion in the study.

\section{Patient characteristics}

A total of 69 patients with stage III Lung Cancer who received treatment in our institution were enrolled, including 34 patients in the HT group and 35 patients in the 3D-CRT group. All patients were staged using 
the TNM 7th edition by American Joint Committee on Cancer (AJCC) and received platinum-based definitive chemoradiotherapy. Patients with older age, severe multiple co-morbidities, higher level of lymph node metastases, large tumor volumes, and centrally located tumors were suggested to receive HT in order to reduce higher dose volume in their normal lungs and OARs. Targeted agents such as EGFR tyrosine kinase inhibitors (TKI) or anaplastic lymphoma kinase $(A L K)$ inhibitors were allowed for adenocarcinoma patients as a maintenance therapy if they had stable or progressive disease. Before receiving the treatment with targeted agents, all adenocarcinoma patients were suggested to undergo a genetic examination, especially for EGFR-mutant or EML4-ALK fusion gene.

\section{Treatment plan and target volume definitions}

Patients were instructed to breathe shallowly during simulation and treatment, and then placed in a supine position, immobilized with a vacuum bag to ascertain the reproducibility and operability. Planning CT scan slices were obtained in the supine position with arms above the head at intervals of $3 \mathrm{~mm}$, from the mandible to the lower edge of the liver. Unlike the 3D-CRT group, fourdimensional computed tomography (4D-CT) simulations were applied in the HT group, to track the motion of the tumors and other internal organs during freebreathing. Target delineations were performed in CMS Focus Treatment Planning System in both groups. The CT images and contours were directly transferred onto the 3D planning system (CMS XiO Treatment Planning System) in the 3D-CRT group and onto the HT Hi-Art II planning system (Accuray) in the HT group, respectively. The pulmonary extent of lung tumors was delineated in pulmonary windows and level settings in CT images, and the mediastinal extent was delineated in mediastinal windows and level settings in CT images. The primary tumor and lymph nodes larger than $1.5 \mathrm{~cm}$ in the short axis in the enhanced CT or FDG-PET/CT were delineated as gross tumor volume (GTV). Clinical target volume (CTV) was defined as GTV plus $6 \mathrm{~mm}$ for squamous cancers and $8 \mathrm{~mm}$ for adenocarcinomas. Clinically uninvolved hilar, mediastinal, and supraclavicular nodal regions were not purposely included in the CTV. Internal target volumes (ITV) which combined the extension of GTVs at the 4 phases of the respiratory cycle on the 4D-CT scan were created. The planning target volume (PTV) extended a margin of $1 \mathrm{~cm}$ from the ITV for patients treated with
3D-CRT and $0.4 \mathrm{~cm}$ for those with HT to account for daily set-up errors. ${ }^{18}$ FDG-PET-guided radiotherapy planning was optional. Image guidance was performed prior to each treatment.

The OARs included the lungs, esophagus, heart, and spinal cord. The total doses of both HT and 3D-CRT were prescribed to the PTV with a minimum $95 \%$ coverage of the reference volume. In the planning process, we focused on a V20 for lungs of $\leq 30 \%$ (acceptable up to $35 \%$ ) and mean lung doses (MLD; both lungs excluding GTV) of $\leq 17$ Gy (acceptable up to 20 Gy), and a maximum dose (Dmax) of 54 Gy to the spinal cord. The relative volume of the esophagus receiving $\geq 55$ Gy did not exceed $30 \%$, and the relative volume of heart that received $\geq 40$ Gy did not exceed $50 \%$.

\section{Follow-up and evaluation}

Patients were assessed after completion of radiotherapy at 4-6 weeks for the first time, and then were followed up every three months for the first two years and every six months thereafter. Clinical examinations and chest CT for toxicity grading and response evaluation were performed at every follow-up. FDG-PET was optional and mainly performed when tumor relapse was suspected. PET were repeated every six months. All acute toxicities were graded according to Common Terminology Criteria for Adverse Events version 4.0 (CTCAE 4.0). Late toxicities were scored using the RTOG late radiation morbidity scoring.

Toxicity mainly centered on radiation pneumonitis (RP), esophagitis, and hematological toxic effects. Acute toxicity lasting for 90 days since the start of radiotherapy was monitored every week during the radiotherapy, while late toxicity was defined as toxicity occurring more than 90 days after radiotherapy.

Disease responses to radiotherapy were evaluated according to the Response Evaluation Criteria in Solid Tumors guideline 1.1 (16) and the following categories were used. Complete response (CR): disappearance of all target lesions for four weeks at least. Partial response (PR): at least $30 \%$ decrease in the sum of the longest diameters of target lesions. Progressive disease (PD): progression in target disease of $20 \%$ increase in sum or a $5 \mathrm{~mm}$ absolute increase when the total sum is very small or detection of new lesions. Stable disease (SD): neither sufficient shrinkage to qualify for PR nor sufficient increase to qualify for PD. Pathological nodes which are defined as measurable and may be identified as target lesions must 
Table 1 Tumor and patient characteristics

\begin{tabular}{|c|c|c|c|}
\hline Characteristic & $\mathrm{HT}(\mathrm{n}=34)$ & 3D-CRT $(n=35)$ & $\mathrm{P}$ \\
\hline Sex & & & 0.428 \\
\hline Female & 2 & 5 & \\
\hline Male & 32 & 30 & \\
\hline \multicolumn{4}{|l|}{ Age, years } \\
\hline$\geq 65$ & 17 & 9 & 0.037 \\
\hline$<65$ & 17 & 26 & \\
\hline PS (ECOG) & & & 0.543 \\
\hline $0-1$ & 23 & 26 & \\
\hline 2 & 11 & 9 & \\
\hline COPD & & & 0.069 \\
\hline Yes & 22 & 15 & \\
\hline No & 12 & 20 & \\
\hline Tumor location & & & 0.537 \\
\hline Central & 20 & 18 & \\
\hline Peripheral & 14 & 17 & \\
\hline Histology & & & 0.272 \\
\hline SCC & 20 & 25 & \\
\hline Adenocarcinoma & 14 & 10 & \\
\hline T stage & & & 0.109 \\
\hline $\mathrm{T} 1-2$ & 15 & 9 & \\
\hline T3-4 & 19 & 26 & \\
\hline N stage & & & 0.010 \\
\hline No-2 & 16 & 27 & \\
\hline N3 & 18 & 8 & \\
\hline Clinical stage & & & 0.271 \\
\hline IIIA & 13 & 18 & \\
\hline IIIB & 21 & 17 & \\
\hline Chemoradiotherapy & & & 0.035 \\
\hline Concurrent & 11 & 4 & \\
\hline Sequential & 23 & 31 & \\
\hline
\end{tabular}

$\mathrm{HT}$, helical tomotherapy; 3D-CRT, three dimensional conformal radiation therapy; PS, performance status; ECOG, Eastern Cooperative Oncology Group; COPD, chronic obstructive pulmonary diseases; SCC, squamous-cell carcinoma.

meet the criterion of a short axis $\geq 15 \mathrm{~mm}$ by CT scan. All other pathological nodes (those with short axis $\geq 10 \mathrm{~mm}$ but $<15 \mathrm{~mm}$ ) were considered non-target lesions. Nodes that had an axis $<10 \mathrm{~mm}$ were considered nonpathological and not to be recorded or followed. The short axis measurement was included in the sum of lesions in calculation of tumour response. Nodes that shrank to a $<10 \mathrm{~mm}$ short axis were considered normal. Local control was defined when the disease responses of pre-treatment primary tumors were assessed CR, PR or SD.

\section{Statistical analyses}

Data are expressed as the mean \pm standard deviation. OS was calculated from initial diagnosis to death due to any cause or to the last follow-up. Local control and PFS were measured from the start of radiotherapy to the first event or to the last follow-up. Chi-square test and Mann-Whitney $\mathrm{U}$ test were used to compare the baseline characteristics and responses to the treatment. The survival date was evaluated by Kaplan-Meier method and the log-rank test was used to determine the presence of a statistical difference between the two groups with respect to survival or disease progression. Univariate and multivariate analyses of survival were performed using the Cox proportional hazards model. All statistical analyses were performed using the SPSS 19.0 biostatistical software package (IBM SPSS, Chicago, IL, USA). A two-sided $\mathrm{P}$ value of $<0.05$ was considered statistically significant in all tests.

\section{Results}

\section{Patients and tumor characteristics}

Demographics and clinical characteristics of both groups are listed in Table 1 . The number of patients $\geq 65$ years were significantly lower in the 3D-CRT group than in the HT group $(\mathrm{P}=0.037)$. Significantly fewer patients with N3 stage were in the 3D-CRT group compared with the HT group $(\mathrm{P}=0.010)$. Significantly fewer patients received concurrent chemoradiotherapy in the $3 \mathrm{D}-\mathrm{CRT}$ group compared with the HT group $(\mathrm{P}=0.035)$. The amount of patients with COPD were slightly fewer in the 3D-CRT group than in the HT group, but not significant $(\mathrm{P}=0.069)$. No other significant differences were found between the two groups.

\section{Treatment of radiotberapy characteristics}

The total dose was 60.0 Gy delivered in 26 days in the HT group and 60.0 Gy delivered in 40 days in the 3D-CRT group. The biologically effective dose (BED) for the present fractionation schedule was estimated using the time-adjusted formula described by Mehta et al. (17). BED = nd $[1+\mathrm{d} /(\alpha / \beta)]-$ $\ln 2$ (T-Tk), where $\mathrm{n}$ is the number of fractions, $\mathrm{d}$ is the dose 
Table 2 Dosimetric parameters

\begin{tabular}{lccc}
\hline Parameter & HT (n=34) & 3D-CRT $(\mathrm{n}=35)$ & $\mathrm{P}$ \\
\hline Lung dose, V2.5 (\%) & $65.0(26.0-92.0)$ & $62.0(24.0-92.0)$ & 0.881 \\
Lung dose, V5 (\%) & $55.0(22.0-66.0)$ & $51.0(24.0-85.0)$ & 0.819 \\
Lung dose, V10 (\%) & $39.5(15.0-53.0)$ & $38.0(17.0-68.0)$ & 0.264 \\
Lung dose, V20 (\%) & $23.0(8.0-46.0)$ & $28.0(7.0-63.0)$ & 0.005 \\
Lung dose, V30 (\%) & $13.0(5.0-41.0)$ & $20.0(0-44.0)$ & 0.001 \\
Lung dose, V40 (\%) & $6.5(0-30.0)$ & $10.0(0-44.0)$ & 0.004 \\
Lung dose, V50 (\%) & $3.0(0-20.0)$ & $3.0(0-28.0)$ & 0.903 \\
Lung dose, V60 (\%) & $0.5(0-16.0)$ & $0(0-9.0)$ & 0.104 \\
Lung MLD (Gy) & $6.0 \pm 3.5$ & $10.3 \pm 4.2$ & $0.000^{*}$ \\
S.C max (Gy) & $31.0 \pm 6.2$ & $36.1 \pm 8.1$ & $0.005^{*}$ \\
S.C mean dose (Gy) & $19.6 \pm 5.8$ & $20.3 \pm 8.3$ & $0.722^{*}$ \\
PTV (cc) & $87.6 \pm 83.4$ & $97.1 \pm 61.2$ & $0.589^{*}$ \\
\hline
\end{tabular}

*, Student's $t$ test; Mann-Whitney test for all other analyses. Vx, percentage volume of lung tissue outside the PTV exposed to $\geq x$ Gy; MLD, mean lung dose; S.C, spinal cord; PTV, planning target volume.

Table 3 Acute and late toxicities

\begin{tabular}{lcccccccccc}
\hline \multirow{2}{*}{ Toxicity } & \multicolumn{3}{c}{$\mathrm{HT}(\mathrm{n}=34)(\%)$} & & \multicolumn{3}{c}{ 3D-CRT $(\mathrm{n}=35)(\%)$} \\
\cline { 2 - 6 } \cline { 8 - 10 } & G1 & G2 & G3 & G4 & & G1 & G2 & G3 & G4 \\
\hline Acute RP & 52.9 & 32.4 & 11.7 & 0 & & 42.9 & 37.1 & 17.1 & 0 \\
Acute RE & 52.9 & 20.6 & 0 & 0 & & 28.6 & 40.0 & 5.7 & 0 \\
Acute MS & & & & & & & & \\
HGB & 2.9 & 0 & 0 & 0 & & 8.6 & 2.9 & 2.9 & 0 \\
WBC & 0 & 8.8 & 0 & 0 & & 2.9 & 20.0 & 0 & 5.7 \\
N & 2.9 & 0 & 0 & 0 & & 5.7 & 0 & 0 & 2.9 \\
PLT & 2.9 & 0 & 0 & 2.9 & 0 & 0 & 0 & 2.9 \\
Late RP & 44.1 & 23.5 & 23.5 & 0 & & 48.6 & 25.7 & 25.7 & 0 \\
Late RE & 0 & 5.9 & 2.9 & & 8.6 & 22.8 & 2.9 & 0 \\
Late MS & & & & & & & & \\
HGB & 0 & 0 & 0 & 0 & & 5.7 & 0 & 2.9 & 0 \\
WBC & 0 & 8.8 & 0 & 0 & & 2.9 & 2.9 & 2.9 & 0 \\
N & 2.8 & 0 & 0 & 0 & & 5.7 & 5.7 & 0 & 0 \\
PLT & 0 & 0 & 0 & 2.8 & 0 & 0 & 0 & 0 \\
\hline
\end{tabular}

HT, helical tomotherapy; 3D-CRT, three dimensional conformal radiation therapy; RP, radiation pneumonitis; RE, radiationesophagitis; MS, myelosuppression (hematological toxic effects); WBC, white blood cell; N, neutrophile; RBC, red blood cell; HGB, hemoglobin; PLT, platelet. per fraction, $\mathrm{T}$ is the overall treatment time, the $\alpha / \beta$ ratio for lung cancer is $10 \mathrm{~Gy}$, with proliferation time starting at 28 days (Tk) (18). The median BED delivered in the HT group was 78.7 Gy and the overall treatment time was 28 days. The median BED delivered in the 3D-CRT group was 69.5 Gy and the overall treatment time was 40 days.

\section{Dose distribution}

Dosimetric parameters and dose distribution for $\mathrm{HT}$ and 3D-CRT groups are summarized in Table 2. V20 ( $\mathrm{P}=0.005)$, V30 $(\mathrm{P}=0.001)$ and $\mathrm{V} 40(\mathrm{P}=0.004)$ were significantly smaller in the HT group than in the 3D-CRT group, whereas V2.5, V5, V10, V50, V60 of the lungs showed no significant difference. Lung MLD was significantly lower in the HT group than in the $3 \mathrm{D}-\mathrm{CRT}$ group $(\mathrm{P}<0.0001)$. Mean dose of spinal cord showed no significant difference between the two groups, whereas max dose of spinal cord was significantly lower in the HT group than in the 3D-CRT group $(\mathrm{P}=0.005)$. PTV showed no significant difference between the two groups.

\section{Toxicity}

In the HT and 3D-CRT groups, grade 2 acute RP was observed in $11(32.4 \%)$ and $13(37.1 \%)$ patients, and grade 3 acute RP was observed in $4(11.8 \%)$ and $6(17.1 \%)$ patients, respectively (Table 3). No patients suffered from grade 4 or 5 acute RP. There was no significant difference in the incidences of acute $\mathrm{RP} \geq$ grade 2 between the two groups $(\mathrm{P}=0.398)$. A patient of 74 years in the HT group receiving sequential chemoradiotherapy, died of respiratory insufficiency caused by a combination effect of an in-field acute grade 3 pneumonitis, pulmonary infection, and persistent tumor. Late $\mathrm{RP} \geq$ grade 2 was observed in $16(47.0 \%)$ and $18(51.4 \%)$ patients in the HT and 3D-CRT groups, respectively, which did not differ significantly between the two groups $(\mathrm{P}=0.717)$. In the HT and 3D-CRT groups, grade 2 acute radiation esophagitis (RE) was observed in 7 (20.6\%) and 14 $(40.0 \%)$ patients, and grade 3 acute RE was observed in $0(0 \%)$ and $2(5.7 \%)$ patients, respectively (Table 3). The incidences of acute $\mathrm{RE} \geq$ grade 2 was significantly lower in the HT group than in the 3D-CRT group ( $\mathrm{P}=0.027)$. Two patients experienced grade 3 acute $\mathrm{RE}$ with weight 
Table 4 Overall response rates after radiotherapy

\begin{tabular}{lcccc}
\hline $\begin{array}{l}\text { Time after } \\
\text { radiotherapy }\end{array}$ & $\begin{array}{c}\text { All patients } \\
(\mathrm{n}=69, \%)\end{array}$ & $\begin{array}{c}\mathrm{HT} \\
(\mathrm{n}=34, \%)\end{array}$ & $\begin{array}{c}\text { 3D-CRT } \\
(\mathrm{n}=35, \%)\end{array}$ & $\mathrm{P}$ \\
\hline 3-month & 79.7 & 88.2 & 71.4 & 0.083 \\
6-month & 62.3 & 70.6 & 54.3 & 0.162 \\
1-year & 42.0 & 52.9 & 31.4 & 0.070 \\
2-year & 30.4 & 44.1 & 17.1 & 0.015 \\
\hline
\end{tabular}

HT, helical tomotherapy; 3D-CRT. three dimensional conformal radiation therapy.

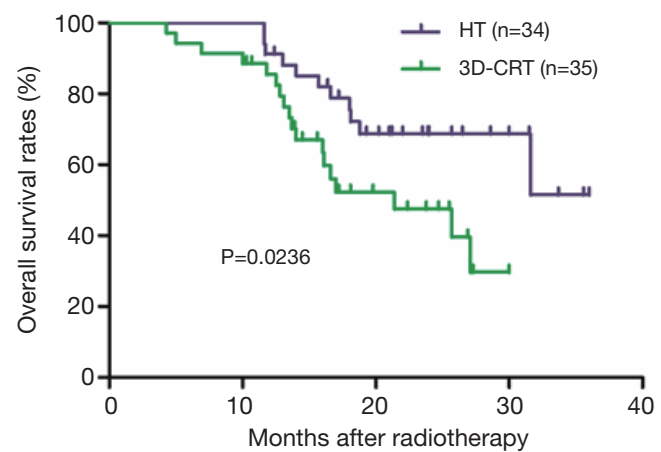

Figure 1 Comparison of overall survival rates in the HT and 3D-CRT groups. The overall survival rate was significantly higher in the HT group than in the 3D-CRT group $(\mathrm{P}=0.0236)$. HT, helical tomotherapy; 3D-CRT, three dimensional conformal radiation therapy.

loss of $\geq 15 \%$ in the $3 \mathrm{D}$-CRT group. One patient relieved soon after the completion of radiotherapy while the other patient developed grade 3 late esophagitis. Grade $\geq 3$ acute esophagitis was not observed in the HT group. Late esophageal toxicity was generally mild. One patient in the HT group with a paraesophageal tumor developed a fatal hemoptysis and died of an esophagotracheal fistula at 13 months after the start of radiotherapy, while no serious acute esophagitis was observed. The patient received a total dose of 60 Gy with hypofractionated (3 Gy/fraction) after two cycles of chemotherapy (gemcitabine and cisplatin). Hematological toxicity rates did not differ significantly between the two groups. Fatigue, chest pain, and skin toxicity were infrequent in both groups.

\section{Overall response and survivals}

Overall response rate $(\mathrm{ORR}=\mathrm{CR}+\mathrm{PR})$ is an evaluation of the short-term treatment efficacy. Three-month, 6-month,

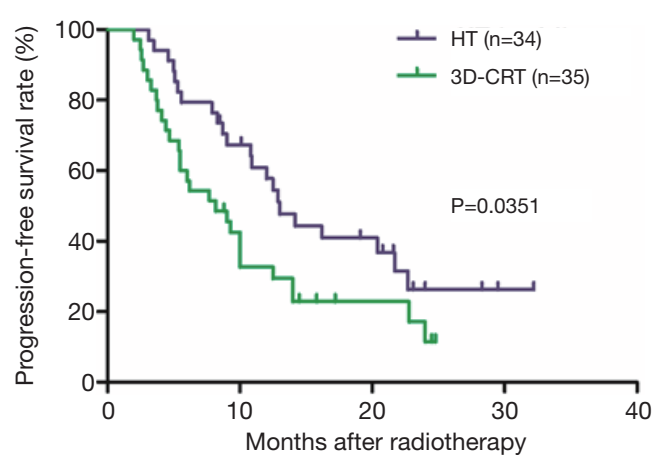

Figure 2 Comparison of progression-free survival rates in the HT and 3D-CRT groups. The progression-free survival rate was significantly higher in the HT group than in the 3D-CRT group ( $\mathrm{P}=0.0351)$. HT, helical tomotherapy; 3D-CRT, three dimensional conformal radiation therapy.

1-year, 2-year ORR in the HT group vs. in the 3D-CRT group were $88.2 \%, 70.6 \%, 52.9 \%, 44.1 \%$ vs. $71.4 \%$, $54.3 \%, 31.4 \%, 17.1 \%$, respectively (Table 4). Three-month, 6-month, 1-year ORR were slightly but insignificantly higher in the HT group than in the $3 \mathrm{D}-\mathrm{CRT}$ group ( $\mathrm{P}=0.083,0.162,0.070$, respectively). Two-year ORR was significantly higher in the HT group than in the $3 \mathrm{D}$-CRT group $(\mathrm{P}=0.015)$.

Among all patients, 1-, and 2-year OS rates were 88.2\% and $58.1 \%$, respectively. These were significantly higher in the HT group (95.0\% and $68.7 \%$, respectively) than in the 3D-CRT group (85.5\% and 47.6\%, respectively; $\mathrm{P}=0.0236$; Figure 1). One-, and 2-year PFS rates were significantly higher in the HT group (57.8\% and $26.3 \%$, respectively) than in the 3D-CRT group $32.7 \%$ and $11.4 \%$, respectively; $\mathrm{P}=0.0351$; Figure 2). Patients in the HT group had a median OS and PFS of 31.6 and 13.0 months, respectively, whereas patients in the 3D-CRT group had a median OS and PFS of 21.4 and 8.2 months, respectively.

\section{Prognostic factors for overall survival (OS) and progression-free survival (PFS)}

Univariate analysis indicated that $\mathrm{PS}(\mathrm{P}=0.011)$, $\mathrm{T}$ stage $(\mathrm{P}=0.047)$ and $\mathrm{RT}$ technique $(\mathrm{P}=0.028)$ were significant prognostic factors of $\mathrm{OS}$ and multivariate analysis indicated that PS $(\mathrm{P}=0.000)$ and RT technique $(\mathrm{P}=0.002)$ were independent prognostic factors of OS (Table 5). Similarly, univariate analysis indicated that $\mathrm{PS}(\mathrm{P}=0.006)$, T stage $(\mathrm{P}=0.018)$ and $\mathrm{RT}$ technique $(\mathrm{P}=0.039)$ were significant prognostic factors of PFS and multivariate analysis indicated 
Table 5 Univariate and multivariate Cox proportional hazards regression analysis for OS and PFS

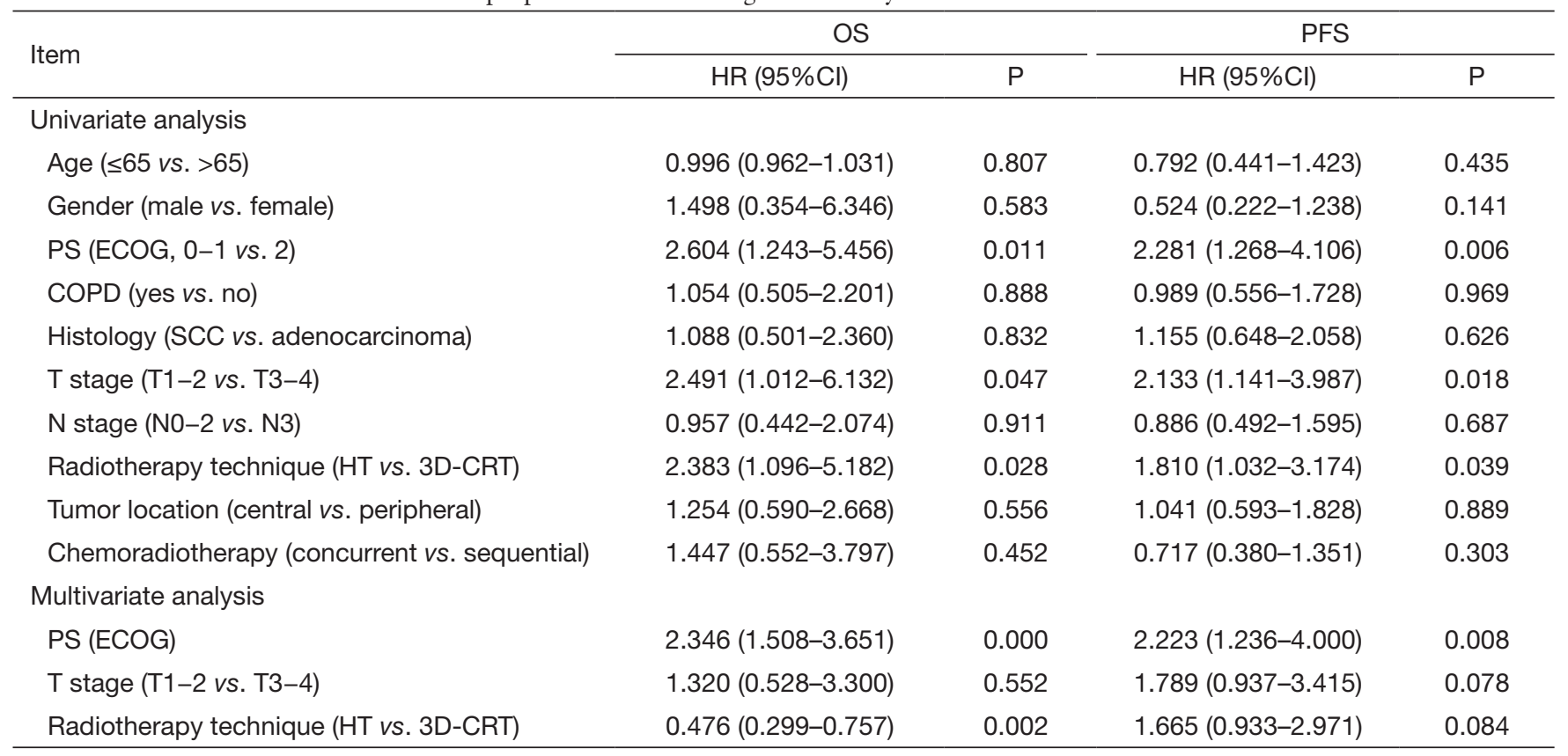

OS, overall survival; PFS, progression-free survival; $\mathrm{Cl}$, confidence interval; HR, hazard ratio; COPD, chronic obstructive pulmonary diseases; PS, performance status; ECOG, Eastern Cooperative Oncology Group; SCC, squamous-cell carcinoma.

that PS $(\mathrm{P}=0.008)$ was an independent prognostic factor of PFS (Table 5).

\section{Discussion}

The standard treatment for locally advanced NSCLC is concurrent chemoradiotherapy. However, poor outcomes (low tumor local control rate and high risk of distant disease spread) were consistently seen in patients with locally advanced disease over a long period of time. RTOG 0617 had tried to escalate total dose to 74 Gy in an attempt to improve outcomes, but the results were surprisingly disappointing that no superior survival was observed in the group with 74 Gy via conventional radiotherapy approaches. Deaths related to pulmonary or cardiopulmonary dysfunction due to high-dose delivered by 3D-CRT and IMRT are the most likely explanations for the outcomes of the RTOG 0617 (19). It can be reasonably assumed that more advanced radiotherapy technologies should be employed when high dose of radiation are applied to patients with locally advanced NSCLC, which brings us to the HT, a novel platform that has already shown the good ability in OARs sparing and can define the target more accurately in various sizes (20-22).
We preformed this study for a direct comparison between hypofractionated radiotherapy at 3 Gy/fraction via $\mathrm{HT}$ with chemotherapy and conventional radiotherapy at $2 \mathrm{~Gy} /$ fraction via 3D-CRT with chemotherapy in stage III NSCLC. Compared with the 3D-CRT group, locoregional and local control survival were significantly better in HT group without increased toxicities, which may indicate hypofractionated RT at $3 \mathrm{~Gy} /$ fraction via HT with chemotherapy is safe and effective.

Although more patients received concurrent chemoradiotherapy in the HT group than in the 3D-CRT group $(32.4 \%$ vs. $11.4 \%, \mathrm{P}=0.035)$, the toxicities were not increased in HT group. In this study, $32.4 \%(11 / 34)$ vs. $37.1 \%(13 / 35)$ and $11.7 \%(4 / 34)$ vs. $17.1 \%(6 / 35)$ of patients had grade 2 and 3 acute RP in the HT vs. 3D-CRT groups, respectively. The incidences of grade 2 and 3 late pneumonitis were $23.5 \%(8 / 34)$ and $23.5 \%$ (8/34) for patients treated with HT, compared with $25.7 \%$ (9/35) and $25.7 \%$ (9/35) for those treated with 3D-CRT. The results are comparable to those reported in the previous literatures (23-25). Eight hundred and thirty-six patients treated with concurrent chemoradiotherapy for NSCLC were analysed in a systematic review by Palma et al. (25). In their analyses, the median total dose delivered was 60 Gy, mostly (73\%) in 
fractionated doses of 2 Gy per day via 3D-CRT or IMRT. The overall rate of grade 2 or higher pneumonitis was $29.8 \%$ (249/836), with fatal pneumonitis in $1.9 \%$. The rate of $\mathrm{RP} \geq$ grade 2 in that study was remarkably lower than ours in 3D-CRT group. Pneumonitis in their analysis might be underestimated. One reason may be that patients who did not receive a full dose of radiation due to development of toxicity during treatment remained included in the study, which may have biased their outcomes. The other reason might be due to the large difference in sample sizes between their study and ours.

It has been reported that elderly patients $(>65 \mathrm{y})$ who undergo carboplatin-paclitaxel chemotherapy are at highest risk of pneumonitis (25). In our study, a 74-year-old patient, receiving sequential paclitaxel chemotherapy with radiotherapy of daily doses of 3 Gy for central location with left upper lobe, died of respiratory insufficiency caused by a combination effect of an in-field acute grade 3 pneumonitis, pulmonary infection and persistent tumor in the HT group. Therefore, $3 \mathrm{~Gy} /$ fraction via HT delivered to old patient with stage III central NSCLC, who may receive carboplatin/paclitaxel, still calls for caution and prudence.

The incidence of grade $\geq 2$ acute esophagitis were $20.6 \%$ and $45.7 \%$ in the HT and 3D-CRT groups, respectively. The incidences of acute $\mathrm{RE} \geq$ grade 2 was significantly lower in the HT group than in the 3D-CRT group $(\mathrm{P}=0.027)$. Our results were in line with previous studies $(26,27)$. Grade $\geq 2$ esophagitis was seen in $40 \%$ of patients with NSCLC in a phase I dose escalation trial of proton beam therapy (27). All patients were treated with hypofractionated (3-4 Gy/fraction) via proton beam therapy without concurrent chemotherapy. Two patients $(8 \%)$ experienced grade 3 esophagitis, one of whom experienced fatal hemoptysis and died of an esophagotracheal fistula, and no recurrence was reported. A fatal esophagotracheal fistula was also seen in our study. Pre-radiation poor PS ( $\geq$ ECOG 2), TLV5, CLV5, and CLV10 were the main determinants of the risk of treatment-related death (26). Our study showed central NSCLC with N3 staging treated with 3D-CRT tended to have a higher incidence of esophagitis.

Compared with the 3D-CRT group, patients in the HT group showed superiority in 2-year ORR. Our findings were in good agreement with other planning studies $(28,29)$. We found a strong association between PFS and ECOG 0-1 performance, the advanced radiotherapy technique. An RTOG analysis has reported that LRC and survival is associated with higher BED among patients who received chemoradiotherapy (29). Another study of
1,390 patients by Machtay et al. evaluated the association between locoregional control (LRC) and survival (3). In line with our outcomes, their analysis revealed that ECOG score, concurrent chemoradiotherapy, and BED were associated with better LRC. They also found that LRC was significantly associated with OS $(\mathrm{P}<0.0001)$ on univariate and multivariate analyses. Overall radiotherapy time was considered as a crucial factor affecting the outcome of radiotherapy in the management of NSCLC, so did total radiation dose (30). RTOG 9410 has highlighted that dose escalation delivered over a standard overall radiotherapy time does not improve OS. The benefits of dose escalation might be realized when overall radiotherapy time is reduced to mitigate accelerated tumor repopulation while advanced radiotherapy techniques are utilized to reduce dose to OARs and thus widening the therapeutic window (31). Therefore, the long overall radiotherapy time and serious toxicity might account for the failure of dose escalation (74 Gy) in RTOG 0617. In our study, compared with the 3D-CRT group, the median BED was escalated to 78.7 Gy and median overall radiotherapy time was reduced to 26.0 days for patients in the HT group which led to better local and locoregional control.

The study found 1- and 2-year OS rates were significantly higher in the HT group (95.0\% and $68.7 \%$, respectively) than in the 3D-CRT group (85.5\% and $47.6 \%$, respectively; $\mathrm{P}=0.0236$ ). Univariate analysis indicated that $\mathrm{PS}$, $\mathrm{T}$ stage and radiotherapy technique were significant prognostic factors for OS. For locally advanced stage NSCLC, it has been demonstrated that improved local tumor control could lead to improved OS despite the high risk of systemic spread (2). The median survival time (MST) of 25.7 months for patients in the 3D-CRT group in our analyses was in line with recent studies, such as the MST of 25.9 months in RTOG 0117 and 28.7 months in the standard dose (60 Gy/30 fractions) group in RTOG 0617. Long-term survival in the HT group is encouraging. The explanation might be that higher dose intensity was received in-field without increasing dose intensity out-of-field in HT group, and more patients receiving treatment with targeted agents as a maintenance therapy during the course of disease in the HT group.

Considering the limitations of sample size and followup time for this study, we support the warnings of RTOG 0617 that high dose (74 Gy) in stage III NSCLC with concurrent chemotherapy need prudence and caution even via HT. A total dose of 60 Gy combined with concurrent chemotherapy should still be the standard treatment for stage III NSCLC. 


\section{Conclusions}

Hypofractionated chemoradiotherapy via HT can shorten the radiotherapy time without increasing treatmentrelated toxicity. The preliminary finding is that OS can be improved by hypofractionated chemoradiotherapy via HT for patients with stage III NSCLC.

\section{Acknowledgements}

None.

\section{Footnote}

Conflicts of Interest: The authors have no conflicts of interest to declare.

\section{References}

1. Siegel R, Naishadham D, Jemal A. Cancer statistics, 2013. CA Cancer J Clin 2013;63:11-30.

2. Aupérin A, Le Péchoux C, Rolland E, et al. Meta-analysis of concomitant versus sequential radiochemotherapy in locally advanced non-small-cell lung cancer. J Clin Oncol 2010;28:2181-90.

3. Machtay M, Paulus R, Moughan J, et al. Defining local-regional control and its importance in locally advanced non-small cell lung carcinoma. J Thorac Oncol 2012;7:716-22.

4. Kong FM, Ten Haken RK, Schipper MJ, et al. High-dose radiation improved local tumor control and overall survival in patients with inoperable/unresectable non-small-cell lung cancer: long-term results of a radiation dose escalation study. Int J Radiat Oncol Biol Phys 2005;63:324-33.

5. Bradley JD, Paulus R, Komaki R, et al. Standard-dose versus high-dose conformal radiotherapy with concurrent and consolidation carboplatin plus paclitaxel with or without cetuximab for patients with stage IIIA or IIIB non-smallcell lung cancer (RTOG 0617): a randomised, two-by-two factorial phase 3 study. Lancet Oncol 2015;16:187-99.

6. Osti MF, Agolli L, Valeriani M, et al. Image guided hypofractionated 3-dimensional radiation therapy in patients with inoperable advanced stage non-small cell lung cancer. Int J Radiat Oncol Biol Phys 2013;85:e157-63.

7. Christodoulou M, Bayman N, McCloskey P, et al. New radiotherapy approaches in locally advanced non-small cell lung cancer. Eur J Cancer 2014;50:525-34.

8. Guckenberger M, Kavanagh A, Partridge M. Combining advanced radiotherapy technologies to maximize safety and tumor control probability in stage III non-small cell lung cancer. Strahlenther Onkol 2012;188:894-900.

9. Baumann M, Herrmann T, Koch R, et al. Final results of the randomized phase III CHARTWEL-trial (ARO 97-1) comparing hyperfractionated-accelerated versus conventionally fractionated radiotherapy in non-small cell lung cancer (NSCLC). Radiother Oncol 2011;100:76-85.

10. Jaffray DA. Image-guided radiotherapy: from current concept to future perspectives. Nat Rev Clin Oncol 2012;9:688-99.

11. Mackie TR, Balog J, Ruchala K, et al. Tomotherapy. Semin Radiat Oncol 1999;9:108-17.

12. Hong TS, Welsh JS, Ritter MA, et al. Megavoltage computed tomography: an emerging tool for image-guided radiotherapy. Am J Clin Oncol 2007;30:617-23.

13. Zelefsky MJ, Kollmeier M, Cox B, et al. Improved clinical outcomes with high-dose image guided radiotherapy compared with non-IGRT for the treatment of clinically localized prostate cancer. Int J Radiat Oncol Biol Phys 2012;84:125-9.

14. Martin JM, Bayley A, Bristow R, et al. Image guided dose escalated prostate radiotherapy: still room to improve. Radiat Oncol 2009;4:50.

15. Lee Y, Auh SL, Wang Y, et al. Therapeutic effects of ablative radiation on local tumor require $\mathrm{CD} 8+\mathrm{T}$ cells: changing strategies for cancer treatment. Blood 2009;114:589-95.

16. Eisenhauer EA, Therasse P, Bogaerts J, et al. New response evaluation criteria in solid tumours: revised RECIST guideline (version 1.1). Eur J Cancer 2009;45:228-47.

17. Mehta M, Scrimger R, Mackie R, et al. A new approach to dose escalation in non-small-cell lung cancer. Int J Radiat Oncol Biol Phys 2001;49:23-33.

18. Roberts SA, Hendry JH. A realistic closed-form radiobiological model of clinical tumor-control data incorporating intertumor heterogeneity. Int J Radiat Oncol Biol Phys 1998;41:689-99.

19. Cox JD. Are the results of RTOG 0617 mysterious? Int J Radiat Oncol Biol Phys 2012;82:1042-4.

20. Servagi Vernat S, Ali D, Puyraveau M, et al. Is IMAT the ultimate evolution of conformal radiotherapy? Dosimetric comparison of helical tomotherapy and volumetric modulated arc therapy for oropharyngeal cancer in a planning study. Phys Med 2014;30:280-5.

21. Yu M, Lee JH, Jang HS, et al. A comparison of dosimetric parameters between tomotherapy and three-dimensional conformal radiotherapy in rectal cancer. Radiat Oncol 
2013;8:181.

22. Chi A, Ma P, Fu G, et al. Critical structure sparing in stereotactic ablative radiotherapy for central lung lesions: helical tomotherapy vs. volumetric modulated arc therapy. PLoS One 2013;8:e59729.

23. Yom SS, Liao Z, Liu HH, et al. Initial evaluation of treatment-related pneumonitis in advanced-stage nonsmall-cell lung cancer patients treated with concurrent chemotherapy and intensity-modulated radiotherapy. Int J Radiat Oncol Biol Phys 2007;68:94-102.

24. Bral S, Duchateau M, Versmessen H, et al. Toxicity and outcome results of a class solution with moderately hypofractionated radiotherapy in inoperable Stage III non-small cell lung cancer using helical tomotherapy. Int J Radiat Oncol Biol Phys 2010;77:1352-9.

25. Palma DAx, Senan S, Tsujino K, et al. Predicting radiation pneumonitis after chemoradiation therapy for lung cancer: an international individual patient data meta-analysis. Int J Radiat Oncol Biol Phys 2013;85:444-50.

26. Song CH, Pyo H, Moon SH, et al. Treatment-related pneumonitis and acute esophagitis in non-small-cell lung cancer patients treated with chemotherapy and helical tomotherapy. Int J Radiat Oncol Biol Phys 2010;78:651-8.

27. Gomez DR, Gillin M, Liao Z, et al. Phase 1 study of dose

Cite this article as: He J, Huang Y, Chen Y, Shi S, Ye L, Hu Y, Zhang J, Zeng Z. Feasibility and efficacy of helical intensity-modulated radiotherapy for stage III non-small cell lung cancer in comparison with conventionally fractionated 3D-CRT. J Thorac Dis 2016;8(5):862-871. doi: $10.21037 /$ jtd.2016.03.46 escalation in hypofractionated proton beam therapy for non-small cell lung cancer. Int J Radiat Oncol Biol Phys 2013;86:665-70.

28. Sher DJ, Koshy M, Liptay MJ, et al. Influence of conformal radiotherapy technique on survival after chemoradiotherapy for patients with stage III non-small cell lung cancer in the National Cancer Data Base. Cancer 2014;120:2060-8.

29. Machtay M, Bae K, Movsas B, et al. Higher biologically effective dose of radiotherapy is associated with improved outcomes for locally advanced non-small cell lung carcinoma treated with chemoradiation: an analysis of the Radiation Therapy Oncology Group. Int J Radiat Oncol Biol Phys 2012;82:425-34.

30. Machtay M, Hsu C, Komaki R, et al. Effect of overall treatment time on outcomes after concurrent chemoradiation for locally advanced non-small-cell lung carcinoma: analysis of the Radiation Therapy Oncology Group (RTOG) experience. Int J Radiat Oncol Biol Phys 2005;63:667-71.

31. Bayman N, Blackhall F, McCloskey P, et al. How can we optimise concurrent chemoradiotherapy for inoperable stage III non-small cell lung cancer? Lung Cancer 2014;83:117-25. 